Theorem 3 and the Lemma give precisely the amount of uniformity required to obtain two sequences of pushes $\phi^{1}, \phi^{2}, \cdots$ and $\psi^{1}$, $\psi^{2}, \cdots$ of $(Q, f(M)$ (converging to $\epsilon / 2$-pushes $\phi$ and $\psi$ of $(Q, f(M))$, respectively, such that $\phi f=\psi g$. The desired $\epsilon$-push of $(Q, f(M))$ is the composition $h=\psi^{-1} \phi$.

Complete proofs of these results will appear in full elsewhere.

Added in proof. Since this announcement was submitted, some errors in the proof of Homma's approximation theorem have been discovered. Should these discrepancies not be rectified, then the restriction $q-m \geqq 3$ must be replaced by $m \leqq(2 / 3) q-1$ in the statements of Theorem 1 and the corollaries.

\title{
REFERENCES
}

1. J. L. Bryant and C. L. Seebeck, III, Locally nice embeddings of polyhedra, Quart. J. Math. Oxford Ser., (to appear).

2. H. Gluck, Embeddings in the trivial range, Ann. of Math. (2) 81 (1965), 195210.

3. J. P. Hempel and D. R. McMillan, Jr., Locally nice embeddings of manifolds, Amer. J. Math. 88 (1966), 1-19.

4. T. Homma, On the imbedding of polyhedra in manifolds, Yokohama Math. J. 10 (1962), 5-10.

5. - Piecewise linear approximations of embeddings of manifolds, Mineographed notes, Florida State University, 1965.

6. J. F. P. Hudson, Concordance and isotopy of PL embeddings, Bull. Amer. Math. Soc. 72 (1966), 534-535.

7. D. R. McMillan, Jr., A criterion for cellularity in a manifold, Ann. of Math. (2) 79 (1964), 327-337.

Florida State University, and

Michigan State University

\section{ERRATUM, VOLUME 73}

Index to Volume 73, p. 1004.

Line 29 should read:

Kahn, Peter J. Chern numbers and oriented homotopy type, 932 